\title{
Novel Potent Inhibitors of Plasmodium vivax Dihydrofolate Reductase: An in silico Antimalarial Drug Discovery
}

\author{
Singh Pushpendra', Prem Prakash Kushwaha², Kumar Shashank ${ }^{2 \star}$ \\ ${ }^{1}$ Tumor Biology Department, National Institute of Pathology, New Delhi, INDIA. \\ ${ }^{2}$ Department of Biochemistry and Microbial Sciences, School of Basic and Applied Sciences, Central University of Punjab, Bathinda, \\ Punjab, INDIA.
}

\begin{abstract}
Objectives: In the present study, we targeted the dihydrofolate reductase enzyme that catalyzes the reduction of dihydrofolate to tetrahydrofolate which is required for the purines and pyrimidine synthesis. Malaria is one of the severe diseases throughout the world caused by blood-borne parasite Plasmodium vivax. Materials and Methods: Eightyfive parthenin analogs were docked against $P$. vivax and Homo sapiens dihydrofolate reductase proteins (PDB 2BL9 and 1KMS respectively) by using Maestro 9.6 program to evaluate the binding affinities of ligands with the protein. Results and Discussion: Docking analysis revealed some best hit ligands against $P$. vivax such as CID3467446 and CID56671343 but not inhibited the mammalian dihydrofolate reductase. The Dock score of parthenin analogs ranged from -7.31 to -9.3 while for standard dihydrofolate reductase inhibitors it was -4.78 to -8.04 . Structural analysis of docked complexes of selected parthenin like compounds with $P$. vivax and mammalian dihydrofolate reductase revealed the involvement of Arg 115, Leu 136, Lys 138, Gly 175, Ser 117, Gln 177 and Ile 7, Ala 9, Thr 56, Ile 60, Pro 61 amino acid residues respectively in strong interactions. Absorption, distribution, metabolism, and excretion properties of best-docked compounds were predicted using QikProp application of Maestro 9.6. The results indicated that all the best-docked lead compounds followed Lipinski's rule of five. Conclusion: Based on the results of the present study it has been concluded that parthenin like compounds may serve as potent dihydrofolate reductase inhibition based anti-malarial drug lead.
\end{abstract}

Key word: Dihydrofolate reductase (DHFR), Malaria, Parthenin analogs (like compounds), Maestro 9.6, Antimalarial Drugs.

\section{INTRODUCTION}

Malaria is a life-threatening disease caused by Plasmodium parasites transmitted to people through malaria vectors. About three million peoples die and five million have been reported to be infected with malaria annually worldwide. ${ }^{1}$ Absence of any effective malarial vaccine, chemotherapy plays crucial role in containment of the disease but unfortunately, drug-resistant strains of Plasmodium such as $P$. vivax have appeared against most of antimalarial introduced till date. Thus increased efforts in antimalarial drug discovery are urgently needed. The goal must be to develop safe and affordable new drugs to counter the spread of malaria parasites that are resistant to existing agents. The malaria parasite resides primarily with in the host erythrocyte, where it exploits host cell components to meet its needs for life-cycle development and degrade the haemoglobin content of infected erythrocyte cells leading to anaemia especially in children and pregnant women., ${ }^{2,3}$ Tetrahydrofolate, is a coenzyme involved in amino acid and nucleotide metabolism. In Plasmodium, it can be synthesized either via a de novo or salvage pathway. Dihydrofolate reductase is one of the important folate pathway
Submission Date: 01-06-2017; Revision Date: 06-07-2017; Accepted Date: 11-01-2018

DOI: 10.5530/ijper.52.1.14 Correspondence: Dr. Shashank Kumar, School of Basic and Applied Sciences, Department of Biochemistry and Microbial Sciences, Central University of Punjab, Bathinda, Punjab, 151001, INDIA.

Phone no: 919335647413

E-mail: shashankbiochemau@gmail.com

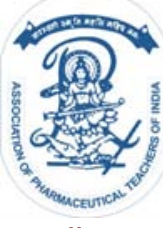

www.ijper.org 


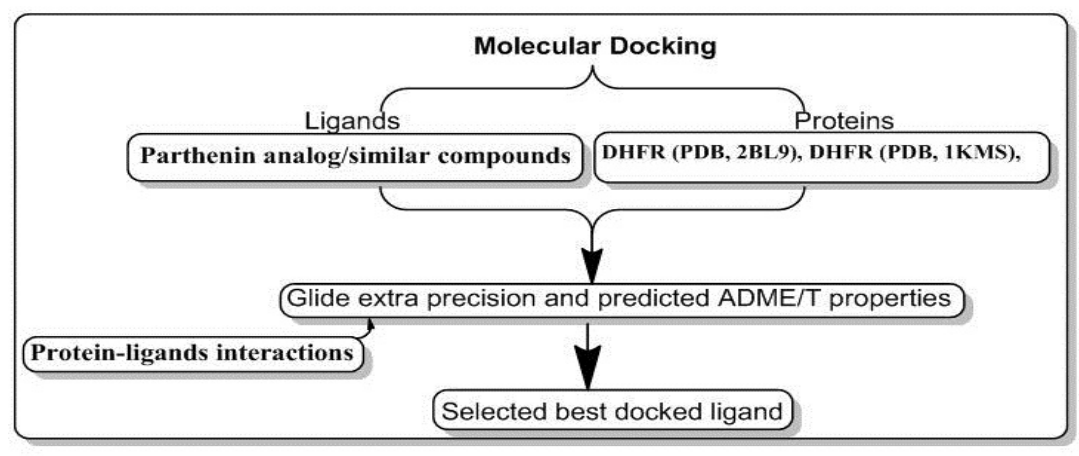

Figure 1: Workflow of study design.

enzyme used as traditional antimalarial targets. Unlike in mammalian cells these enzymes exist in malaria parasites as bi-functional enzymes. It catalyzes the NADPH-dependent reduction of $\mathrm{H}_{2}$ folate to $\mathrm{H}_{4}$ folate, a necessary co-factor for the biosynthesis of thymidylate, purine nucleotides, and certain amino acids. ${ }^{4}$ 1,2,4-Triazole and 2,3-disubstituted quinazoline-4(3H)-one analogs are studies against DHFR and shown that it increases the potential activity against malarial., ${ }^{5,6}$ Some of the most widely used antimalarial drugs inhibit folate metabolism such as sulfonamides and sulfones, usually combined with pyrimethamine or biguanides (DHFR inhibitors). Unfortunately, emergence of resistance against these drugs malaria chemotherapy and prevention has been hampered. It has been reported that resistance is determined by mutations, which do not have the same effect on all antifolates. This offers some hope for development of new DHFR inhibitors. ${ }^{4}$ On the basis of above discussion DHFR may represent a potential target for malaria. Parthenium hysterophorus is a terrestrial weed found all around the world. P. hysterophorus confers many health benefits such as remedy for skin inflammation, rheumatic pain, diarrhoea, urinary tract infections, dysentery and neuralgia. Beside these several studies reported the anti-malarial potential of different parts of this plant. Parthenin is the major active phytoconstituents of P. hysterophorus leaf, stem flower and root. ${ }^{7}$ Due to this we used different analogs of parthenin like compounds to target $P$. vivax DHFR enzyme in the present in silico study. In silico screening approach is the leading technique for preliminary identification of novel inhibitors for target proteins and predicting their biological binding mode. The majorities of anti-malarial drugs are small molecules designed to bind, interact and modulate the biological activity of the different pathogen proteins. Molecular docking inheres of three key consecutive goals; pose prediction, virtual screening and binding affinity evaluation. Computer aided drug designing is being exploited to identify hits, pick leads and optimize leads i.e. transform biologically active compounds into good drugs by enhancing their physicochemical, pharmaceutical and ADME $\backslash \mathrm{T}$ (absorption, distribution, metabolism, excretion and toxicity) properties. Thus in silico modeling is used considerably to minimize risk, time and resource requirements of chemical synthesis and biological in vitro and in vivo testing. ${ }^{8}$ In the present study we used Maestro 9.6 Schrodinger software to dock eighty five parthenin like compounds with malaria parasite and human DHFR protein. Furthermore QikProp application of Maestro 9.6 was used to predict $\mathrm{ADME} / \mathrm{T}$ properties of best-docked analogs.

\section{MATERIALS AND METHODS}

\section{Selection of Ligand and Protein molecules}

GLIDE based molecular docking protocol adapted from our previous published literature with minor modifications. ${ }^{9}$ (Figure 1). Selected ligand dataset for the study are given in Table 1.

PubChem molecules having molecular weight of $<500$ Da were screened in this study. Recommended values of estimated number of hydrogen bonds that would be accepted by the solute from water molecules in an aqueous solution was $<10$. For hydrogen bonds that would be donated by the solute to water molecules in an aqueous solution was $<5$. Averages values are taken over a number of configurations, so they can be non-integer.

All selected ligands were subjected to Ligprep wizard application of the Maestro 9.6 (Schrödinger Inc). Ligprep performs many corrections on the ligands, such as the addition of hydrogens, 2D to 3D conversion, corrected bond lengths and bond angles, low energy structure, stereochemistries and ring conformation. Ten tautomer's generated per ligands by using Maestro 9.6. The X-ray crystal structure of DHFR, PDB: 2BL9.$^{10}$ DHFR PDB: $1 \mathrm{KMS}{ }^{11}$ retrieved from the protein data bank. Maestro 


\begin{tabular}{|c|c|c|c|c|c|c|c|c|c|}
\hline S. No. & Molecule & Mol MW & Dipole & SASA & FOSA & FISA & PISA & D-HB & A-HB \\
\hline 1 & 23275702 & 290.358 & 6.92 & 501.933 & 321.143 & 111.692 & 69.099 & 0 & 5.7 \\
\hline 2 & 23205 & 262.305 & 7.708 & 463.589 & 238.823 & 126.449 & 98.317 & 0 & 5.7 \\
\hline 3 & 52167 & 262.305 & 4.478 & 462.106 & 232.043 & 128.879 & 101.184 & 0 & 5.7 \\
\hline 4 & 88590 & 264.321 & 10.249 & 446.305 & 253.949 & 123.771 & 68.586 & 1 & 5.75 \\
\hline 5 & 92119 & 246.305 & 9.786 & 458.22 & 250.566 & 102.738 & 104.916 & 0 & 5 \\
\hline 6 & 93016 & 262.305 & 5.522 & 463.569 & 234.256 & 132.766 & 96.547 & 0 & 5.7 \\
\hline 7 & 155585 & 278.304 & 3.912 & 460.576 & 211.41 & 178.439 & 70.727 & 0 & 6.4 \\
\hline 8 & 158187 & 248.278 & 6.223 & 433.316 & 191.452 & 141.326 & 100.538 & 1 & 5.75 \\
\hline 9 & 164614 & 262.305 & 4.161 & 459.175 & 249.736 & 137.775 & 71.663 & 0 & 5.7 \\
\hline 10 & 174870 & 320.341 & 9.207 & 539.219 & 276.753 & 181.851 & 80.615 & 1 & 7.75 \\
\hline 11 & 257272 & 246.305 & 9.989 & 465.024 & 256.95 & 106.975 & 101.099 & 0 & 5 \\
\hline 12 & 257275 & 262.305 & 11.279 & 463.21 & 238.093 & 127.294 & 97.823 & 1 & 5.75 \\
\hline 13 & 296217 & 344.407 & 4.834 & 585.451 & 372.161 & 114.347 & 98.943 & 0 & 7 \\
\hline 14 & 442288 & 262.305 & 8.47 & 449.284 & 233.25 & 122.218 & 93.816 & 1 & 5.75 \\
\hline 15 & 3467446 & 320.341 & 8.516 & 565.168 & 304.122 & 168.307 & 92.74 & 1 & 7.75 \\
\hline 16 & 3482906 & 264.321 & 8.831 & 470.388 & 280.4 & 125.557 & 64.431 & 1 & 5.75 \\
\hline 17 & 3482907 & 278.304 & 9.95 & 462.468 & 225.502 & 166.677 & 70.29 & 0 & 6.4 \\
\hline 18 & 5317982 & 262.305 & 4.478 & 462.106 & 232.043 & 128.879 & 101.184 & 0 & 5.7 \\
\hline 19 & 5318381 & 264.321 & 10.365 & 453.183 & 267.181 & 121.166 & 64.835 & 1 & 5.75 \\
\hline 20 & 6325688 & 262.305 & 5.522 & 463.569 & 234.256 & 132.766 & 96.547 & 0 & 5.7 \\
\hline 21 & 6442202 & 344.407 & 4.825 & 542.718 & 319.962 & 132.757 & 89.998 & 0 & 7 \\
\hline 22 & 6610301 & 262.305 & 6.484 & 458.641 & 225.539 & 133.699 & 99.402 & 0 & 5.7 \\
\hline 23 & 6713099 & 262.305 & 6.683 & 461.595 & 231.607 & 130.343 & 99.645 & 0 & 5.7 \\
\hline 24 & 9993590 & 276.332 & 8.721 & 476.867 & 289.33 & 118.946 & 68.592 & 1 & 5.75 \\
\hline 25 & 10265551 & 278.304 & 10.623 & 515.658 & 233.172 & 178.237 & 104.248 & 1 & 7.75 \\
\hline 26 & 10333765 & 278.304 & 8.892 & 508.515 & 241.538 & 159.771 & 107.206 & 1 & 7.75 \\
\hline 27 & 10356188 & 278.304 & 7.518 & 509.188 & 257.618 & 145.348 & 106.222 & 1 & 7.75 \\
\hline 28 & 10400472 & 262.305 & 7.861 & 464.974 & 242.053 & 124.47 & 98.451 & 1 & 5.75 \\
\hline 29 & 10400473 & 262.305 & 6.683 & 461.595 & 231.607 & 130.343 & 99.645 & 0 & 5.7 \\
\hline 30 & 10989149 & 264.321 & 8.373 & 458.962 & 273.685 & 119.058 & 66.219 & 1 & 5.75 \\
\hline 31 & 11302856 & 360.406 & 5.981 & 574.447 & 316.059 & 156.815 & 101.573 & 0 & 7.7 \\
\hline 32 & 11552273 & 362.422 & 3.225 & 593.511 & 369.486 & 130.657 & 93.369 & 0 & 7.7 \\
\hline 33 & 11660489 & 360.406 & 8.882 & 592.841 & 341.166 & 158.948 & 92.727 & 0 & 7.7 \\
\hline 34 & 12310702 & 264.321 & 8.233 & 459.882 & 275.658 & 119.025 & 65.198 & 1 & 5.75 \\
\hline 35 & 13918467 & 278.304 & 5.927 & 531.796 & 251.883 & 174.808 & 105.105 & 1 & 7.75 \\
\hline 36 & 13918470 & 278.304 & 10.507 & 522.974 & 239.158 & 179.699 & 104.117 & 1 & 7.75 \\
\hline 37 & 15139250 & 276.332 & 9.961 & 478.692 & 297.682 & 115.469 & 65.541 & 1 & 5.75 \\
\hline 38 & 15605026 & 278.304 & 10.943 & 471.099 & 226.474 & 174.902 & 69.723 & 1 & 6.45 \\
\hline 39 & 23246961 & 278.304 & 9.728 & 529.319 & 247.196 & 173.708 & 108.415 & 1 & 7.75 \\
\hline 40 & 23246962 & 278.304 & 8.196 & 501.341 & 228.608 & 168.772 & 103.961 & 1 & 7.75 \\
\hline 41 & 23275702 & 290.358 & 6.92 & 501.933 & 321.143 & 111.692 & 69.099 & 0 & 5.7 \\
\hline 42 & 44176867 & 246.305 & 10.032 & 461.265 & 249.069 & 106.944 & 105.253 & 0 & 5 \\
\hline 43 & 44383456 & 262.305 & 7.708 & 463.589 & 238.823 & 126.449 & 98.317 & 0 & 5.7 \\
\hline 44 & 44444898 & 262.305 & 5.523 & 463.569 & 234.256 & 132.766 & 96.547 & 0 & 5.7 \\
\hline 45 & 44468437 & 358.477 & 8.367 & 680.051 & 493.254 & 120.951 & 65.846 & 1 & 5.75 \\
\hline
\end{tabular}

Continued... 


\begin{tabular}{|c|c|c|c|c|c|c|c|c|c|}
\hline 46 & 44470367 & 302.369 & 8.469 & 550.701 & 360.223 & 121.007 & 69.471 & 1 & 5.75 \\
\hline 47 & 44470368 & 316.396 & 10.779 & 587.213 & 407.641 & 120.908 & 58.665 & 1 & 5.75 \\
\hline 48 & 44470369 & 330.423 & 10.65 & 617.058 & 433.821 & 120.831 & 62.406 & 1 & 5.75 \\
\hline 49 & 44567134 & 402.443 & 9.069 & 629.923 & 390.893 & 183.897 & 55.133 & 1 & 10.7 \\
\hline 50 & 44567136 & 388.416 & 6.533 & 608.209 & 355.74 & 190 & 62.469 & 1 & 10.7 \\
\hline 51 & 44583940 & 362.422 & 3.225 & 593.511 & 369.486 & 130.657 & 93.369 & 0 & 7.7 \\
\hline 52 & 53298618 & 262.305 & 11.177 & 465.837 & 233.342 & 132.107 & 100.388 & 1 & 5.75 \\
\hline 53 & 54671714 & 262.305 & 10.985 & 472.249 & 239.476 & 134.128 & 98.645 & 1 & 6.7 \\
\hline 54 & 56664965 & 304.342 & 7.436 & 505.161 & 293.314 & 128.967 & 82.88 & 0 & 7 \\
\hline 55 & 56668390 & 332.396 & 11.477 & 566.921 & 346.447 & 146.726 & 73.747 & 0 & 7 \\
\hline 56 & 56671343 & 292.331 & 9.795 & 502.807 & 276.799 & 174.142 & 51.867 & 1 & 6.45 \\
\hline 57 & 56678611 & 318.369 & 7.035 & 542.355 & 329.609 & 127.455 & 85.292 & 0 & 7 \\
\hline 58 & 58399512 & 262.305 & 11.177 & 465.837 & 233.342 & 132.107 & 100.388 & 1 & 5.75 \\
\hline 59 & 58399517 & 250.294 & 11.103 & 447.74 & 244.603 & 136.881 & 66.256 & 1 & 5.75 \\
\hline 60 & 59513914 & 262.305 & 5.891 & 453.918 & 250.586 & 115.408 & 87.925 & 0 & 5.7 \\
\hline 61 & 68034684 & 262.305 & 3.485 & 446.828 & 216.22 & 133.788 & 96.819 & 0 & 5.7 \\
\hline 62 & 68152825 & 262.305 & 8.894 & 464.129 & 231.588 & 132.214 & 100.326 & 1 & 5.75 \\
\hline 63 & 70498184 & 280.32 & 8.367 & 480.172 & 209.933 & 182.568 & 87.671 & 2 & 5.45 \\
\hline 64 & 70498261 & 262.305 & 4.101 & 458.171 & 223.058 & 130.489 & 104.624 & 0 & 5.7 \\
\hline 65 & 72786361 & 360.406 & 7.921 & 553.412 & 303.906 & 151.158 & 98.347 & 0 & 7.7 \\
\hline 66 & 72791246 & 360.406 & 10.202 & 559.567 & 300.775 & 165.022 & 93.77 & 0 & 7.7 \\
\hline 67 & $b^{*}$ & 362.422 & 6.128 & 583.404 & 343.848 & 151.244 & 88.312 & 0 & 7.7 \\
\hline 68 & $a^{*}$ & 278.304 & 12.34 & 458.286 & 234.221 & 155.985 & 68.079 & 1 & 6.45 \\
\hline 69 & $c^{*}$ & 402.443 & 10.701 & 653.753 & 406.752 & 195.922 & 51.079 & 1 & 10.7 \\
\hline 70 & 75072308 & 388.416 & 8.961 & 629.686 & 368.517 & 190.013 & 71.156 & 1 & 10.7 \\
\hline 71 & 76166589 & 262.305 & 11.317 & 460.569 & 234.575 & 128.355 & 97.639 & 1 & 6.7 \\
\hline 72 & $\mathrm{~d}^{*}$ & 330.423 & 9.017 & 615.948 & 433.453 & 119.777 & 62.718 & 1 & 5.75 \\
\hline 73 & 76390479 & 304.342 & 7.499 & 515.185 & 294.507 & 134.407 & 86.271 & 0 & 7 \\
\hline 74 & 76391109 & 316.396 & 11.205 & 581.684 & 389.334 & 124.617 & 67.734 & 1 & 5.75 \\
\hline 75 & 76391203 & 332.396 & 7.563 & 546.249 & 341.377 & 115.403 & 89.469 & 0 & 7 \\
\hline 76 & $\mathrm{e}^{*}$ & 358.477 & 9.053 & 682.214 & 495.198 & 124.834 & 62.182 & 1 & 5.75 \\
\hline 77 & 76393399 & 318.369 & 11.364 & 538.702 & 336.122 & 109.771 & 92.809 & 0 & 7 \\
\hline 78 & 76776246 & 250.294 & 11.369 & 446.519 & 249.288 & 131.625 & 65.607 & 1 & 5.75 \\
\hline 79 & 77977597 & 280.32 & 3.808 & 458.115 & 201.696 & 168.282 & 88.136 & 2 & 5.45 \\
\hline 80 & 78178185 & 248.278 & 4.2 & 436.341 & 193.611 & 146.186 & 96.543 & 1 & 5.75 \\
\hline 81 & 78178433 & 262.305 & 6.603 & 452.273 & 257.132 & 123.522 & 71.619 & 0 & 5.7 \\
\hline 82 & 78410193 & 344.407 & 5.913 & 575.505 & 362.161 & 118.382 & 94.962 & 0 & 7 \\
\hline 83 & 85132821 & 262.305 & 7.341 & 463.328 & 237.639 & 128.251 & 97.437 & 1 & 5.75 \\
\hline 84 & 90473934 & 262.305 & 10.718 & 460.849 & 231.846 & 128.38 & 100.623 & 1 & 5.75 \\
\hline 85 & 442288 & 262.305 & 8.474 & 460.87 & 231.755 & 128.539 & 100.576 & 1 & 5.75 \\
\hline
\end{tabular}

$\mathrm{a} *(6 \mathrm{R}, 6 \mathrm{aS}, 9 \mathrm{bR})-6 \mathrm{a}$-hydroxy-3-(hydroxymethyl)-6, 9a-dimethyl- $4 \mathrm{H}, 5 \mathrm{H}, 6 \mathrm{H}, 9 \mathrm{bH}$-azuleno[4, 5-b]furan-2,9-dione

$\mathrm{b} *(3 \mathrm{~S}, 3 \mathrm{aS}, 4 \mathrm{~S}, 4 \mathrm{aS}, 7 \mathrm{aR}, 8 \mathrm{~S}, 9 \mathrm{aS})-3,4 \mathrm{a}, 8$-trimethyl-2, 5-dioxo- $3 \mathrm{H}, 3 \mathrm{aH}, 4 \mathrm{H}, 7 \mathrm{aH}, 8 \mathrm{H}, 9 \mathrm{H}, 9 \mathrm{aH}$-azuleno[6, 5-b]furan-4-yl (3S)-3-hydroxy-2-methylidenebutanoate c* (3S, 4S, 9bR)-3-acetyl-6-(hydroxymethyl)-3, 9-dimethyl-2, 7-dioxo-2 $\mathrm{H}, 3 \mathrm{H}, 3 \mathrm{aH}, 4 \mathrm{H}, 5 \mathrm{H}, 7 \mathrm{H}, 8 \mathrm{H}, 9 \mathrm{bH}$ - azuleno[4, 5-b]furan-4-yl (2E)-2-methylbut-2-enoate d* (3aS, 6S, 6aS, 9aR, 9bS)-6a-hydroxy-6, 9a-dimethyl-3-methylidene-8-pentyl-2H, 3H, 3aH, 4H, 5H, 6H, 6aH, 9H, 9aH, 9bH-azuleno[4, 5-b]furan-2,9-dione e* (3aR, 6S, 6aS, 9aR, 9bR)-8-heptyl-6a-hydroxy-6, 9a-dimethyl-3-methylidene- $2 \mathrm{H}, 3 \mathrm{H}, 3 \mathrm{aH}, 4 \mathrm{H}, 5 \mathrm{H}, 6 \mathrm{H}, 6 \mathrm{aH}, 9 \mathrm{H}, 9 \mathrm{H}, 9 \mathrm{bH}$-azuleno[4, 5-b]furan-2,9-dione mol_MW= Molecular Weight of the molecule; SASA= Solvent Accessible Surface Area; FOSA= Hydrophobic component of the SASA (saturated carbon and attached hydrogen); FISA= Hydrophilic component of the SASA (SASA on N, O, H on heteroatoms, and carbonyl C); PISA= $\pi$ (carbon and attached hydrogen) component of the SASA; D-HB= Donar Hydrogen Bond; $\mathrm{A}-\mathrm{HB}=$ Acceptor Hydrogen Bond 
9.6 protein preparation wizard application performed for the correction of raw PDB structure; these are the addition of hydrogen atoms, assigning bond orders and bond length, creation of disulphide bonds, fixing of the charges and orientation of groups were included in to the protein molecules.

\section{Molecular docking}

Molecular docking studies using the chosen ligand molecules were conducted using Maestro 9.6. ${ }^{12}$ Each of these compounds was docked into protein molecules, and the docking conformation possessing the lowest energy was fixed. After preparation of ligands and protein, optimized potential for liquid simulations (OPLS_2005) force field applied for local energy minimization (bond stretching energy) and geometry optimization..$^{13,14,15}$ After the execution OPLS_2005, a receptor-grid file was generated. Van der Waal radii of receptor atom by $1.00 \AA$ and a partial atomic charge of 0.25 scaled for the generation of the receptor grid and consequently molecular docking was performed.

\section{ADME/T properties studies}

The majority of drug doesn't accomplish something in clinical trials due to deprived ADME/T properties. Therefore, in silico ADME/T (Absorption, Distribution, Metabolism, Excretion and Toxicity) predictive tools that could eliminate inappropriate compounds, before invested valuable time and money in primary testing of compounds. Computer based theoretical approaches transpire to be the best option for prediction of ADME/T, for new compounds. Thus, ADME/T properties of best-docked compounds were predicted using QikProp application of Maestro 9.6. ADME/T properties are prerequisite for the drug discovery and development process. ${ }^{16,17}$

\section{RESULTS AND DISCUSSION}

\section{Analysis of docking results of promising compounds for Plasmodium vivax DHFR}

Crystal structure of dihydrofolate reductase from Plasmodium vivax: pyrimethamine displacement linked with mutation-induced resistance in complex with inhibitors have been reported, which provide information about the exact location and composition of inhibitor binding pocket and opportunity to use the enzyme in a functional conformation. ${ }^{10}$ We used X-ray structure of Plasmodium vivax DHFR in complex inhibitor (PDB id code 2BL9) for the docking study. Pyrimethamine (Pyr) targets dihydrofolate reductase of Plasmodium vivax (PvDHFR) as well as other malarial parasites, but its use as antimalarial is hampered by the widespread high resistance. Our selected dihydrofolate reductase of Plasmodium vivax structural insights suggest a general approach for developing new generations of antimalarial DHFR inhibitors that, by only occupying substrate space of the active site, would retain binding affinity with the mutant enzymes. ${ }^{10}$ Molecular docking was performed using the extra precision (XP) mode of grid-based ligand docking with energetics (GLIDE). We also used known DHFR inhibitors for the comparison of results (Table 2, Figure 2). Our result highlighted that; CID3467446, (6R, 6aS, 9bR)-6a-hydroxy-3-(hydroxymethyl)-6, 9a-dimethyl$4 \mathrm{H}, 5 \mathrm{H}, 6 \mathrm{H}, 9 \mathrm{bH}$-azuleno[4, 5-b] furan-2,9-dione, CID3467446-2, CID56671343, (3S, 3aS, 4S, 4aS, 7aR, 8S, 9aS)-3, 4a, 8-trimethyl-2,5-dioxo-3H, 3aH, 4H, 7aH, $8 \mathrm{H}, 9 \mathrm{H}, 9 \mathrm{aH}$-azuleno[6,5-b] furan-4- yl (3S)-3-hydroxy2-methylidenebutanoate yielded a pre-eminent dock score for with proteins Plasmodium vivax DHFR -8.12, $-8.77,-7.52,-7.42,-7.31 \mathrm{Kcal} / \mathrm{mol}$ respectively (Table 2 ). Moreover, these compounds do not have better Gscore against human DHFR. Most of the interactions made by compounds with residues in active site of Plasmodium vivax DHFR seem to be hydrophobic in nature. Proteinligand interactions of 2BL9 with compounds showed that amino acids Leu136, Ile155, and Val178 appeared in the hydrophobic interactions. Furthermore, amino acids Arg115, Leu136, Lus138, and Gly175 involved in back-bone hydrogen bonding of protein-ligand interactions (Figure 3)

\section{Interaction Modes between the parthenin like compounds and human DHFR}

Molecular docking of Plasmodium vivaxDHFR and human DHFR against natural compounds has been carried out. In the present investigation, our result highlighted that; (3aS, 6S, 6aS, 9aR, 9bS)-6a-hydroxy-6, 9a-dimethyl3-methylidene-8-pentyl-2H, 3H, 3aH, 4H, 5H, $6 \mathrm{H}, 6 \mathrm{aH}$, 9H, 9aH, 9bH-azuleno[4, 5-b]furan-2,9-dione, (3aR, 6S, 6aS, 9aR, 9bR)-8-heptyl-6a-hydroxy-6, 9a-dimethyl3-methylidene- $2 \mathrm{H}, 3 \mathrm{H}, 3 \mathrm{aH}, 4 \mathrm{H}, 5 \mathrm{H}, 6 \mathrm{H}, 6 \mathrm{aH}, 9 \mathrm{H}$, 9aH, 9bH-azuleno[4, 5-b]furan-2,9-dione, (3aR, 6S, 6aS, 9aR, 9bR)-8-heptyl-6a-hydroxy-6, 9a-dimethyl-3-methylidene- $2 \mathrm{H}, 3 \mathrm{H}, 3 \mathrm{aH}, 4 \mathrm{H}, 5 \mathrm{H}, 6 \mathrm{H}, 6 \mathrm{aH}, 9 \mathrm{H}, 9 \mathrm{aH}$, 9bH-azuleno[4, 5-b] furan-2,9-dione-2, (3S, 4S, 9bR)3-acetyl-6-(hydroxymethyl)-3, 9- dimethyl-2, 7-dioxo$2 \mathrm{H}, 3 \mathrm{H}, 3 \mathrm{aH}, 4 \mathrm{H}, 5 \mathrm{H}, 7 \mathrm{H}, 8 \mathrm{H}, 9 \mathrm{bH}-$ azuleno[4, 5-b] furan-4-yl (2E)-2-methylbut-2-enoate, (3aR, 6S, 6aS, 9aR, 9bR)-8-heptyl-6a-hydroxy-6, 9a-dimethyl-3-methylidene- $2 \mathrm{H}, 3 \mathrm{H}, 3 \mathrm{aH}, 4 \mathrm{H}, 5 \mathrm{H}, 6 \mathrm{H}, 6 \mathrm{aH}, 9 \mathrm{H}, 9 \mathrm{aH}, 9 \mathrm{bH}-$ azuleno[4, 5-b] furan-2,9-dione -3 yielded a pre-eminent dock score for with proteins human DHFR -9.3, -9.04, $-9.01,-9.03,-8.93 \mathrm{Kcal} / \mathrm{mol}$ respectively (Table 3 ). 


\begin{tabular}{l} 
Table 2: Lowest binding energy for the ligand-DHFR (PDB, 2BL9) protein interaction as detected by GLIDE \\
molecular docking. \\
\hline \multirow{3}{*}{ Ligand type }
\end{tabular}

$\mathrm{a} *(6 \mathrm{R}, 6 \mathrm{aS}, 9 \mathrm{bR})-6 \mathrm{a}$-hydroxy-3-(hydroxymethyl)-6, 9a-dimethyl-4H, 5H, 6H, gbH-azuleno[4, 5-b]furan-2,9-dione

$\mathrm{b}^{*}(3 \mathrm{~S}, 3 \mathrm{aS}, 4 \mathrm{~S}, 4 \mathrm{aS}, 7 \mathrm{aR}, 8 \mathrm{~S}, 9 \mathrm{aS})-3,4 \mathrm{a}, 8$-trimethyl-2, 5-dioxo-3H, 3aH, 4H, 7aH $, 8 \mathrm{H}, 9 \mathrm{H}, 9 \mathrm{aH}$-azuleno[6, 5-b]furan-4-yl (3S)-3-hydroxy-2-methylidenebutanoate
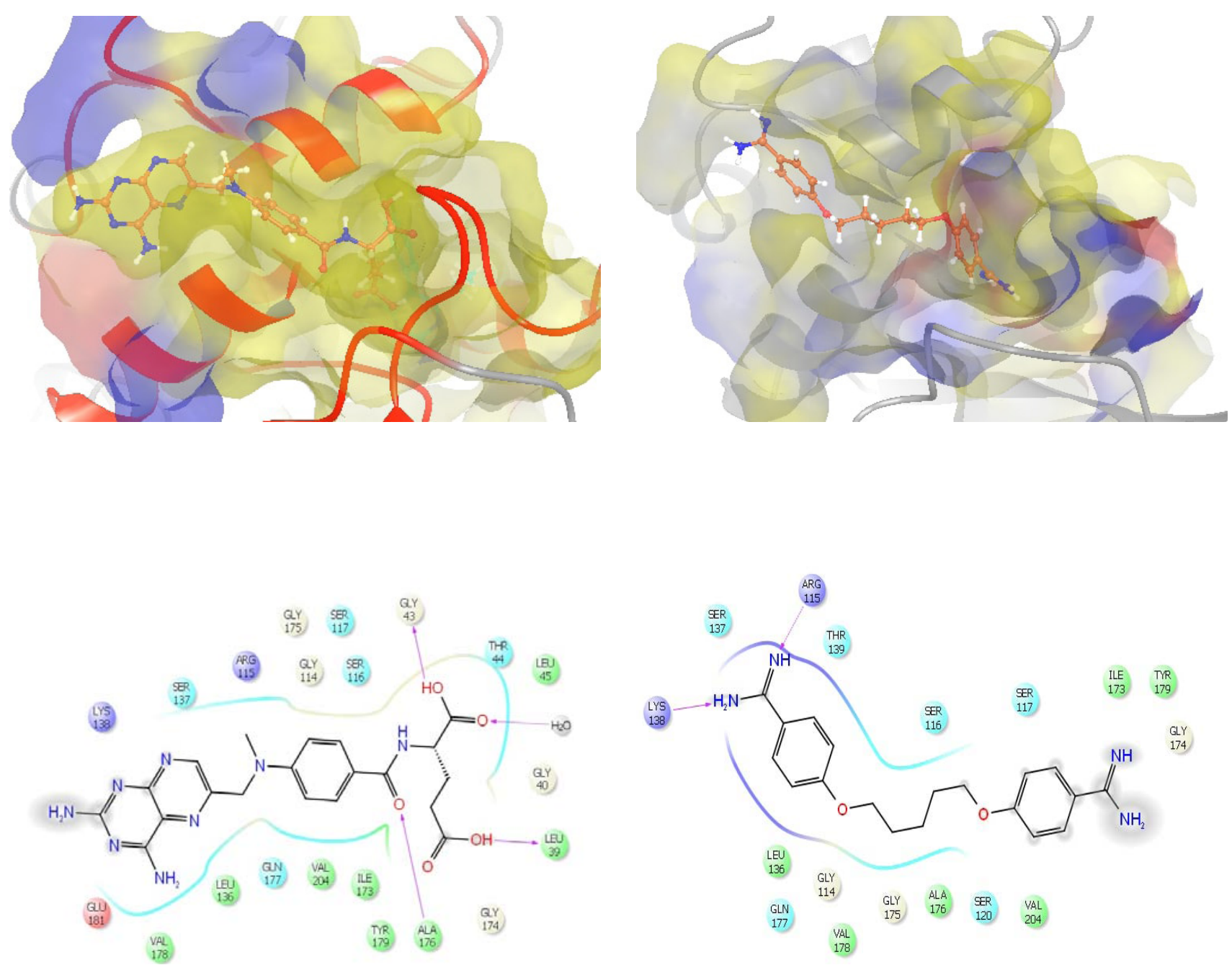

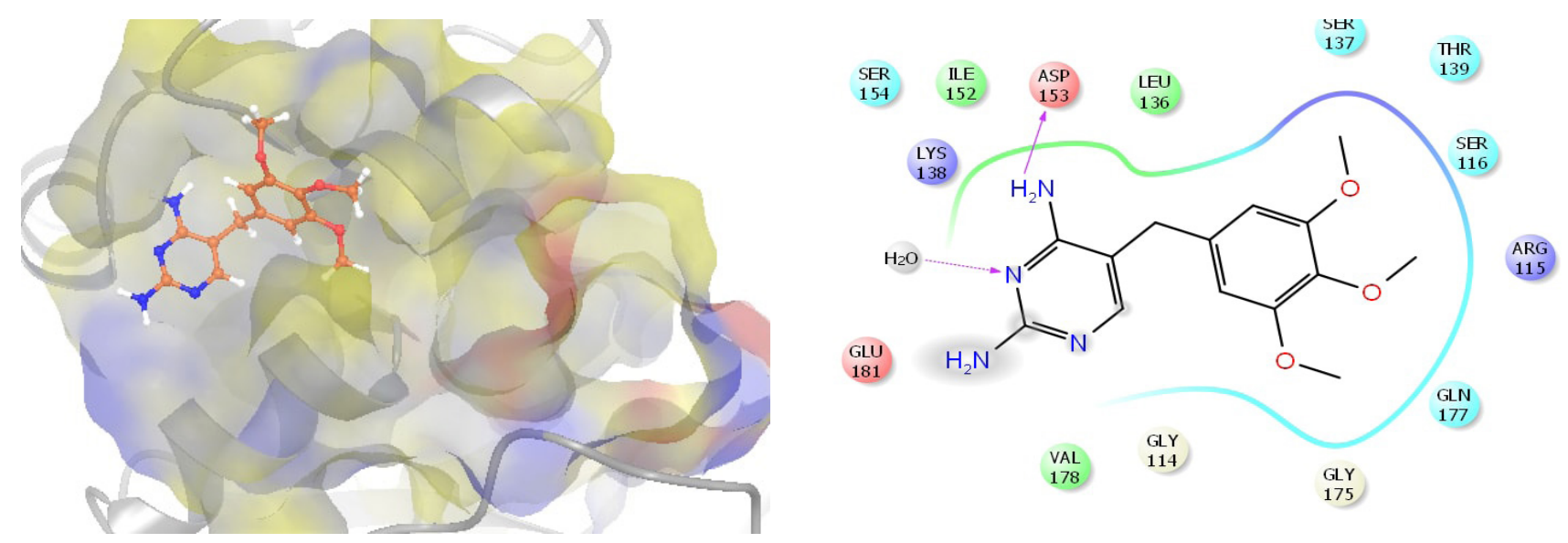

Figure 2: Interaction of $P$. vivax DHFR with standard antimalarial drugs.

(a) Ribbon presentation of DHFR (PDB, 2BL9) protein molecule with CID126941 (b) Protein-ligand interactions profile of 2BL9 with CID126941 (c) Ribbon presentation of DHFR (PDB, 2BL9) protein molecule with CID4735 (d) Protein-ligand interactions profile of 2BL9 with CID4735 (e) Ribbon presentation of DHFR (PDB, 2BL9) protein molecule with CID5578 (f) Protein-ligand interactions profile of 2BL9 with CID5578
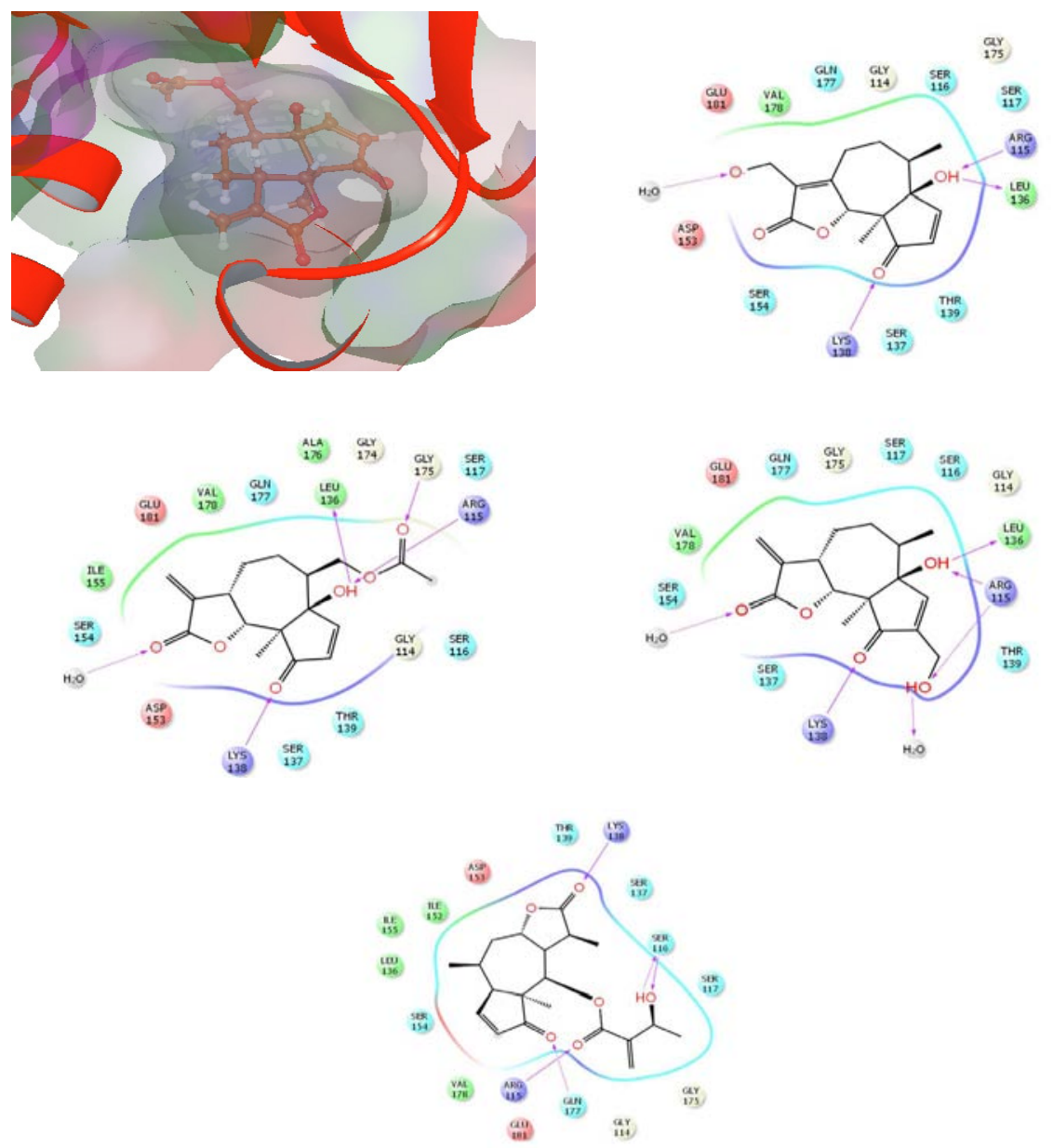

Figure 3: Interaction of $P$. vivax DHFR with parthenin like compounds.

(a) Ribbon presentation of DHFR (PDB, 2BL9) protein molecule with CID3467446 (b) Protein-ligand interactions profile of $2 B L 9$ with CID3467446 (c) Protein-ligand interactions profile of 2BL9 with (6R, 6aS, 9bR)-6a-hydroxy-3-(hydroxymethyl)-6, 9a-dimethyl-4H, 5H, 6H, 9bH-azuleno[4, 5-b]furan-2,9-dione (d) Protein-ligand interactions profile of 2BL9 with CID56671343 (e) Proteinligand interactions profile of 2BL9 with (3S, 3aS, 4S, 4aS, 7aR, 8S, 9aS)-3, 4a, 8-trimethyl-2, 5-dioxo-3H, 3aH, 4H, 7aH, 8H, 9H, 9aH-azuleno[6, 5-b]furan-4- yl (3S)-3-hydroxy-2-methylidenebutanoate 

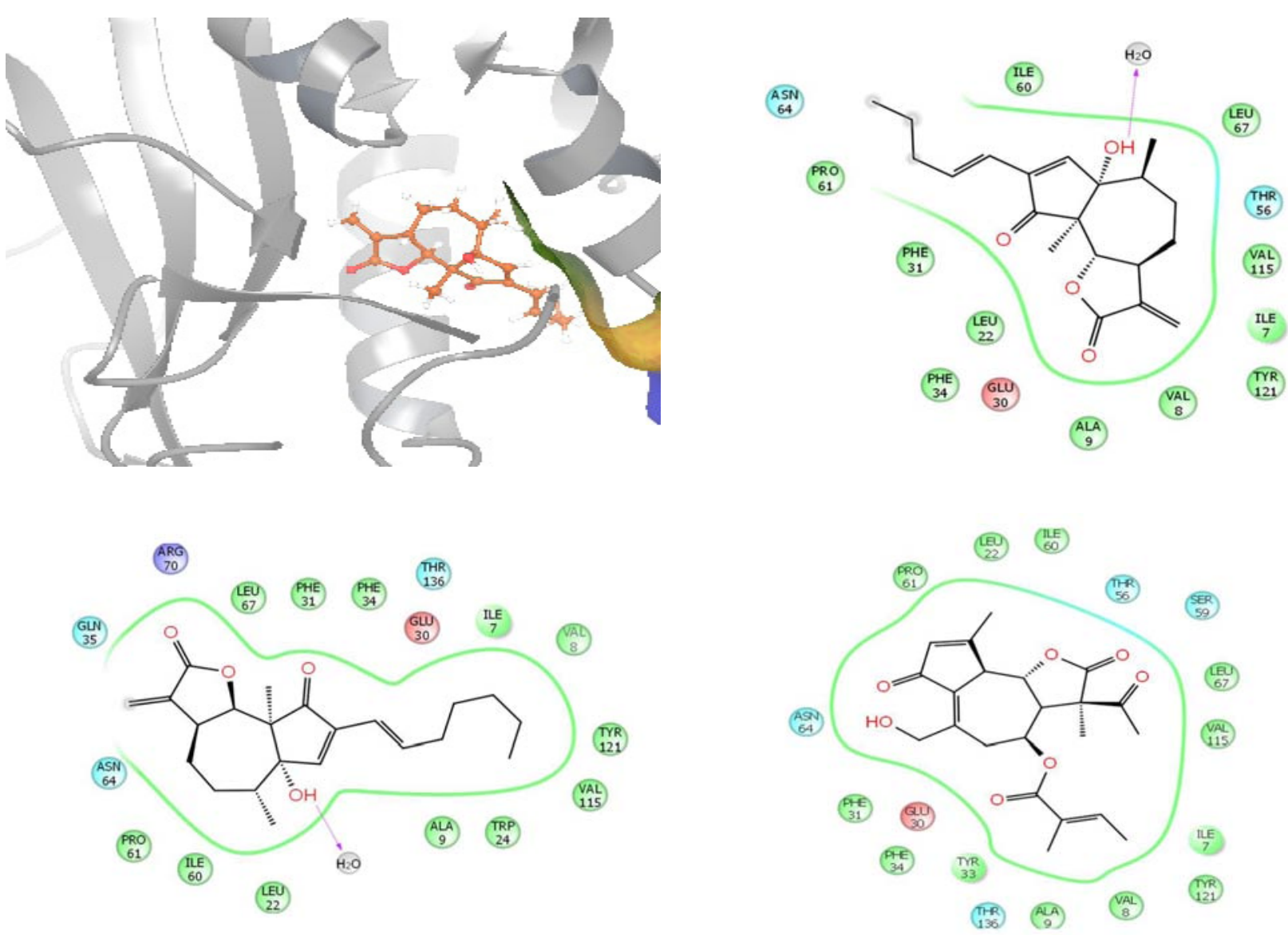

Figure 4: Interaction of Homo sapiens DHFR with parthenin like compounds(a) Ribbon presentation of DHFR (PDB, 1KMS) protein molecule with (3aS, 6S, 6aS, 9aR, 9bS)-6a-hydroxy-6, 9a-dimethyl-3-methylidene-8-pentyl-2H, 3H, 3aH, 4H, 5H, 6H, 6aH, $9 \mathrm{H}, 9 \mathrm{aH}, 9 \mathrm{bH}-a z u l e n o[4,5-\mathrm{b}]$ furan-2,9-dione (b) Protein-ligand interactions profile of 1KMS with (3aS, 6S, 6aS, 9aR, 9bS)-6ahydroxy-6, 9a-dimethyl-3-methylidene-8-pentyl-2H, 3H, 3aH, 4H, 5H, 6H, 6aH, 9H, 9aH, 9bH-azuleno[4, 5-b]furan-2,9-dione (c) Protein-ligand interactions profile of $1 \mathrm{KMS}$ with (3aR, 6S, 6aS, 9aR, 9bR)-8-heptyl-6a-hydroxy-6, 9a-dimethyl-3-methylidene-2H, $3 \mathrm{H}, 3 \mathrm{aH}, 4 \mathrm{H}, 5 \mathrm{H}, 6 \mathrm{H}, 6 \mathrm{aH}, 9 \mathrm{H}, 9 \mathrm{aH}, 9 \mathrm{bH}$-azuleno[4, 5-b]furan-2,9-dione (d) Protein-ligand interactions profile of $1 \mathrm{KMS}$ with (3S, 4S, 9bR)-3-acetyl-6-(hydroxymethyl)-3, 9- dimethyl-2, 7-dioxo-2H, 3H, 3aH, 4H, 5H, 7H, 8H, 9bH- azuleno[4, 5-b]furan-4-yl (2E)2-methylbut-2-enoate.

Protein-ligand interactions delineate that the lipophilic, hydrogen bonding and $\pi-\pi$ stacking interactions represent a central role in protein-ligand interactions at the active site. Molecular docking procedure identifies the docking free energy value (G-score) against these receptor protein molecules. Protein-ligands interactions highlighted the lipophilic, electrostatic and hydrogen bond interactions are a key player in protein-ligand interactions. All the compounds in the dataset were docked into the active site of human DHFR, using the same protocol. Figure 4 depicts the binding conformations of the parthenin like compounds in the binding pocket of the human DHFR. The active site of human DHFR comprises of mostly hydrophobic amino acids as Ile7, Ala9, Trp24, Phe31, Phe34, Thr56, Pro61, and Ile60, Val115 and these amino acid residues are involved in strong hydrophobic interactions with the parthenin like compounds. As expected, inhibitors used in this study bind to the same site like the docked ligand in the crystallographic complex. The inhibitors bind in a hydrophobic pocket adjacent to helix $\alpha \mathrm{B}$, with the 5-deazapteridine ring almost perpendicular to the 5-quinolylamino group. The 5-deazapteridine ring of the inhibitors forms hydrophobic contacts with Val8, Ile7 and Phe31. Notably, the human DHFR parthenin like compound inhibitors displayed same interaction Ile 7, Thr56 and Ile60 knowledgeable by some cycloguanil analogues which proved to be active against influenza virus and respiratory syncytial virus replication, via targeting the host (human) DHFR enzyme. ${ }^{18}$ In another study 5-deazapteridine rings of DMDP (2, 4-diamino5-methyl-5-deazapteridine) derivatives has been shown to bound human DHFR active site in an identical fashion, as reported in case of other inhibitors like methotrexate. ${ }^{19}$ Recently, Viira et al. screened curcuminoids for their in silico antimalarial activity against $P$. falciparum and found 17 potential lead compounds, which they further tested 


\section{Table 3: Lowest binding energy for the ligand-DHFR (PDB, 1KMS) protein interaction as detected by GLIDE} molecular docking.

\begin{tabular}{|c|c|c|c|c|c|c|}
\hline Ligand type & $\begin{array}{c}\text { Compounds } \\
\text { ID }\end{array}$ & GScore & $\begin{array}{l}\text { Lipophilic } \\
\text { Evdw }\end{array}$ & HBond & Electro & Protein-ligands interactions \\
\hline \multirow{10}{*}{ Compounds } & $d^{*}$ & -9.3 & -6.35 & -0.7 & -0.26 & Ile7, Ala9, Thr56, and Ile60 \\
\hline & $e^{*}$ & -9.04 & -6.13 & -0.7 & -0.4 & Ile7, Ala9, Thr56, and Ile60 \\
\hline & $e^{*}-2$ & -9.01 & -6.36 & -0.54 & -0.19 & Ile7, Ala9, Thr56, and Ile60 \\
\hline & $c^{*}$ & -9.03 & -6.04 & -0.48 & 0.04 & Ile7, Thr56, Pro61, and Ile60 \\
\hline & $e^{*}-3$ & -8.93 & -6.23 & -0.5 & -0.24 & Ile7, Thr56, Pro61, and Ile60 \\
\hline & $d^{*}-2$ & -8.82 & -5.76 & -0.7 & -0.43 & Ile7, Thr56, Pro61, and Ile60 \\
\hline & 23275702 & -8.76 & -5.08 & -0.95 & -0.31 & Ile7, Thr56, Pro61, and Ile60 \\
\hline & 3482907 & -8.7 & -4.54 & -1.44 & -0.53 & Ile7, Thr56, Pro61, and Ile60 \\
\hline & 56671343 & -8.6 & -4.68 & -1.44 & -0.36 & Ile7, Thr56, Pro61, and Ile60 \\
\hline & 75072308 & -8.6 & -5.44 & -0.53 & -0.09 & Ile7, Thr56, Pro61, and Ile60 \\
\hline
\end{tabular}

$c^{*}(3 \mathrm{~S}, 4 \mathrm{~S}, 9 \mathrm{bR})-3$-acetyl-6-(hydroxymethyl)-3, 9-dimethyl-2, 7-dioxo-2 $\mathrm{H}, 3 \mathrm{H}, 3 \mathrm{aH}, 4 \mathrm{H}, 5 \mathrm{H}, 7 \mathrm{H}, 8 \mathrm{H}, 9 \mathrm{bH}$ - azuleno[4, 5-b]furan-4-yl (2E)-2-methylbut-2-enoate $\mathrm{d} *(3 \mathrm{aS}, 6 \mathrm{~S}, 6 \mathrm{aS}, 9 \mathrm{aR}, 9 \mathrm{bS})-6 a-h y d r o x y-6$, 9a-dimethyl-3-methylidene-8-pentyl-2 $\mathrm{H}, 3 \mathrm{H}, 3 \mathrm{aH}, 4 \mathrm{H}, 5 \mathrm{H}, 6 \mathrm{H}, 6 \mathrm{aH}, 9 \mathrm{H}, 9 \mathrm{aH}, 9 \mathrm{bH}$-azuleno[4, 5-b]furan-2,9-dione e* (3aR, 6S, 6aS, gaR, 9bR)-8-heptyl-6a-hydroxy-6, 9a-dimethyl-3-methylidene-2H, 3H, 3aH, 4H, 5H, 6H, 6aH, 9H, 9aH, 9bH-azuleno[4, 5-b]furan-2,9-dione Molecule CID; Pubchem IDs, GScore; Glide extra precision scores (kcal/mol)., Lipophilic E Vdw; Chemscore lipophilic pair term and fraction of the total protein-ligand vdw energy., HBond; Hydrogen-bonding term., Electro; Electrostatic rewards., Protein ligands interaction; $\pi-\pi$ stacking, $\pi-$ cat interaction and hydrogen bond between the ligands and protein.

by using in vitro antimalarial assay and found that most of the lead compounds were potential antimalarial agents. $^{20}$

\section{ADME/T properties of leads molecules}

ADME/T properties of lead compounds (Figure 6) were appraised by using the Qikprop application of Maestro 9.621. Most attractive aspect of CID3467446, (6R, 6aS, 9bR)-6a-hydroxy-3-(hydroxymethyl)-6, 9a-dimethyl-4H, 5H, 6H, 9bH-azuleno[4, 5-b]furan2,9-dione, 3467446, 56671343, (3S, 3aS, 4S, 4aS, 7aR, 8S, 9aS)-3, 4a, 8-trimethyl-2, 5-dioxo-3H, 3aH, 4H, 7aH, $8 \mathrm{H}, 9 \mathrm{H}, 9 \mathrm{aH}$-azuleno[6, 5-b]furan-4- yl (3S)-3-hydroxy2-methylidenebutanoate, 3467446, 3482907, 3467446, 3482907, 77977597 are their admirable, Qplogpo/w, QplogHERG, QplogBB, QPP MDCK, Qplogkhsa, and Percentage of human oral value which satisfy the lipinski's rule of five (Table 4).

Moreover, polar surface area, high oral bioavailability, H-bond donors and acceptors are being imperative criteria for the development of therapeutic agents. Veber et al. reported that compounds having 10 or fewer rotatable bonds and polar surface area equal to or less than $140 \AA$ (or 12 or fewer H-bond donors and acceptors) may have a high probability for best oral bioavailability in the rat. ${ }^{22}$ Furthermore, it is also reported that polar surface area is inversely proportional to permeation rate. ${ }^{23}$ These compounds have better SASA values that are claimed to be suitable for therapeutic agents. These results indicate that the compounds will have better penetration rate. Blood Brain Barrier (BBB) involves biochemical barriers such as metabolic enzyme systems and efflux transporters for the xenobiotics. hERG (human Ether-a-go-go-Related Gene) gene codes $\mathrm{K}_{\mathrm{v}} 11.1$ protein ( $\alpha$ subunit of $\mathrm{K}^{+}$channel). It conducts potassium ions out of the heart muscle cells and coordinates the heart's beating. In silico human colon adenocarcinoma (Caco-2) and Madin-Darby canine kidney (MDCK) epithelial cell models are used to evaluate drug's permeability and transporter interactions. Structure-based serum albumin binding model was used to determine the distribution and metabolism of lead compounds. This encompasses the in silico binding strength of lead compounds to human serum albumin. Human oral bioavailability deals with the information about fraction of an administered drug that reaches its site of action through systematic circulation, to exert its pharmacological and therapeutic effects. All these models unscrew the qualitative prediction and ranking of absorption, determining mechanism(s) of permeability, formulation effects on drug permeability, and the potential for transporter-mediated drug-drug interactions.

\section{CONCLUSION}

In this work, molecular docking studies were carried out to explore the binding mechanism of parthenin like compounds derivatives to the $P$. vivax and human DHFR enzyme to enable the design of new parthenin like compound-based human DHFR inhibitors. Both the binding conformation of parthenin like compounds and their binding free energies were predicted by molecular docking. Present study can be considered as an in silico 


\section{Table 4: Structural, physicochemical, biochemical, pharmacokinetics and toxicity properties of compound.}

\begin{tabular}{|c|c|c|c|c|c|c|c|c|}
\hline S.N. & Molecule & $\begin{array}{l}\text { QP log } \\
P_{\text {o/w }} \\
(-2.0 \\
\text { to 6.5) }\end{array}$ & $\begin{array}{l}\text { QPlog HERG } \\
\text { (acceptable } \\
\text { range: above } \\
-5.0 \text { ) }\end{array}$ & $\begin{array}{l}\text { QPP Caco } \\
\text { (nm/sec) } \\
<25-\text { poor } \\
>500 \text { - great }\end{array}$ & $\begin{array}{c}\text { QP log } \\
\text { BB } \\
(-3-1.2)\end{array}$ & $\begin{array}{c}\text { QPP } \\
\text { MDCK } \\
\text { (nm/sec) } \\
<25-\text { poor } \\
>500 \text { - great }\end{array}$ & $\begin{array}{c}\text { QPlog Khsa } \\
\text { (Acceptable } \\
\text { range: }-1.5 \text { to } \\
1.5) .\end{array}$ & $\begin{array}{c}\text { Percentage } \\
\text { of human ora } \\
\text { absorption; } \\
\text { (<25\% is poo } \\
\text { and }>80 \% \text { is } \\
\text { high) }\end{array}$ \\
\hline 1 & 3467446 & 1.023 & -4.076 & 150.058 & -1.362 & 63.677 & -0.353 & 71.888 \\
\hline 2 & $a^{*}$ & 0.865 & -3.382 & 258.352 & -0.974 & 114.557 & -0.391 & 75.182 \\
\hline 3 & 3467446 & 0.965 & -4.163 & 121.167 & -1.469 & 50.536 & -0.351 & 69.886 \\
\hline 4 & 56671343 & 1.251 & -3.48 & 265.101 & -1.062 & 117.795 & -0.274 & 77.646 \\
\hline 5 & $b^{*}$ & 1.56 & -3.611 & 339.947 & -0.953 & 154.121 & -0.507 & 81.39 \\
\hline 6 & 3467446 & 0.962 & -4.026 & 150.658 & -1.348 & 63.952 & -0.377 & 71.558 \\
\hline 7 & 3482907 & 0.574 & -2.87 & 297.89 & -0.833 & 133.619 & -0.808 & 74.588 \\
\hline 8 & 3467446 & 0.947 & -3.855 & 170.727 & -1.259 & 73.208 & -0.395 & 72.447 \\
\hline 9 & 3482907 & 0.54 & -3.279 & 218.621 & -1.014 & 95.639 & -0.78 & 71.985 \\
\hline 10 & 77977597 & 1.76 & -1.282 & 53.449 & -1.039 & 26.536 & -0.339 & 68.179 \\
\hline
\end{tabular}

$\mathrm{a} *(6 \mathrm{R}, 6 \mathrm{aS}, 9 \mathrm{bR})-6 \mathrm{a}-\mathrm{hydroxy}-3$-(hydroxymethyl)-6, 9a-dimethyl- $4 \mathrm{H}, 5 \mathrm{H}, 6 \mathrm{H}, 9 \mathrm{bH}$-azuleno[4, 5-b]furan-2,9-dione

b* (3S, 3aS, 4S, 4aS, 7aR, 8S, 9aS)-3, 4a, 8-trimethyl-2, 5-dioxo-3H, 3aH, 4H, 7aH, 8H, 9H, 9aH-azuleno[6, 5-b]furan-4-yl (3S)-3-hydroxy-2-methylidenebutanoate QPlogPo/w (-2.0 to 6.5) Predicted octanol/water partition coefficient

QPlogHerg (acceptable range: above -5.0) Predicted IC 50 value for blockage of HERG K+ channels;

OPPCaco $(\mathrm{nm} / \mathrm{sec})<25$-poor $>500$ - great Predicted apparent Caco- 2 cell permeability in $\mathrm{nm} / \mathrm{sec}$. Caco- 2 cells is a model for the gut blood barrier,

QPlogBB (-3-1.2) Predicted brain/blood partition coefficient;

OPPMDCK (nm/sec) <25-poor > 500-great Predicted apparent MDCK cell permeability in $\mathrm{nm} / \mathrm{sec}$. MDCK cells are considered to be a good mimic for the blood-brain barrier. Q P log Khsa-Prediction of binding to human serum albumin; (acceptable range: -1.5 to 1.5 ).

Percentage of human oral absorption; ( $<25 \%$ is poor and $>80 \%$ is high)

approach to search for novel class of Dihydrofolate reductase (Plasmodium vivax and human) inhibitors using parthenin like compounds as a scaffold. Results obtained from molecular property analysis clearly indicate that some of the selected parthenin like compounds satisfies criteria's of Lipinski's rule of five, hence has potential to be utilized as effective anti-DHFR agents. The tetrahydrofolate synthesis and DHFR inhibition by parthenin like compounds represented in Figure 5.

Results from docking investigation of selected compounds on Plasmodium vivax DHFR point out that compound CID3467446, (6R, 6aS, 9bR)-6a-hydroxy-3-(hydroxymethyl)-6, 9a-dimethyl-4H, 5H, 6H, 9bH-azuleno[4, 5-b]furan-2,9-dione, 3467446-2, 56671343, (3S, 3aS, 4S, 4aS, 7aR, 8S, 9aS)-3, 4a, 8-trimethyl-2, 5-dioxo-3H, 3aH, $4 \mathrm{H}, 7 \mathrm{aH}, 8 \mathrm{H}, 9 \mathrm{H}, 9 \mathrm{aH}-a z u l e n o[6,5-\mathrm{b}]$ furan-4- yl (3S)-3hydroxy-2-methylidenebutanoate can potentially be used as starting lead compound in developing anti-DHFR agents for Plasmodium vivax. Moreover, compound ( $3 \mathrm{aS}$, 6S, 6aS, 9aR, 9bS)-6a-hydroxy-6, 9a-dimethyl-3-methylidene-8-pentyl- $2 \mathrm{H}, 3 \mathrm{H}, 3 \mathrm{aH}, 4 \mathrm{H}, 5 \mathrm{H}, 6 \mathrm{H}, 6 \mathrm{aH}, 9 \mathrm{H}$, 9aH, 9bH-azuleno[4, 5-b] furan-2,9-dione, (3aR, 6S, 6aS, 9aR, 9bR)-8-heptyl-6a-hydroxy-6, 9a-dimethyl-3-methylidene- $2 \mathrm{H}, 3 \mathrm{H}, 3 \mathrm{aH}, 4 \mathrm{H}, 5 \mathrm{H}, 6 \mathrm{H}, 6 \mathrm{aH}, 9 \mathrm{H}, 9 \mathrm{aH}$, 9bH-azuleno[4,5-b]furan-2,9-dione, and (3S, 4S, 9bR)-

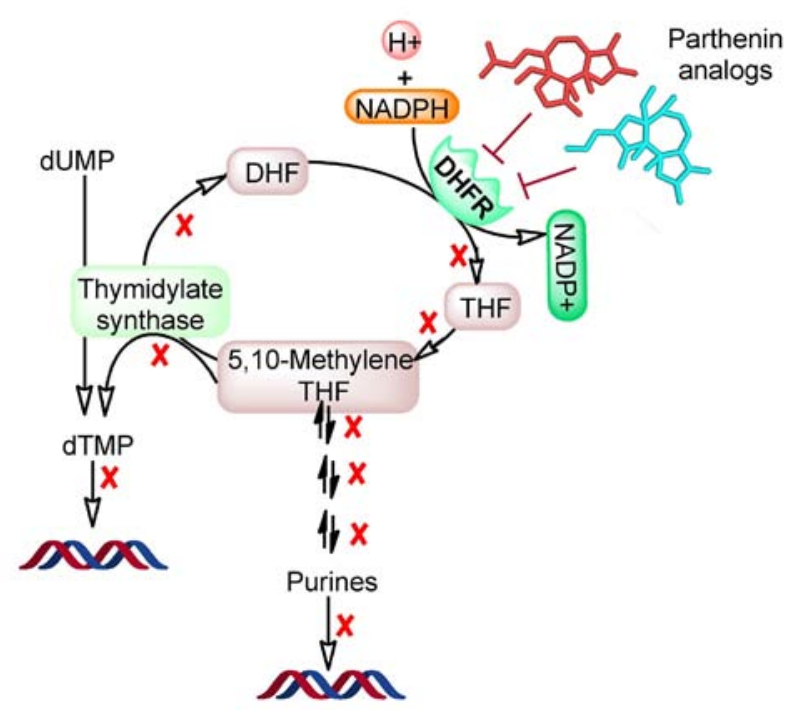

Figure 5: Parthenin like compounds inhibit dihydrofolate reductase (folate pathway) in $P$. vivax.

3-acetyl-6-(hydroxymethyl)-3, 9- dimethyl-2, 7-dioxo$2 \mathrm{H}, 3 \mathrm{H}, 3 \mathrm{aH}, 4 \mathrm{H}, 5 \mathrm{H}, 7 \mathrm{H}, 8 \mathrm{H}, 9 \mathrm{bH}-$ azuleno[4, 5-b] furan-4-yl (2E)-2-methylbut-2-enoate can potentially be used as starting lead compound in developing antiDHFR agents for mammals. 
<smiles>C=C1C(=O)OC2(C)C1CCC(COC(C)=O)C1(O)C=CC(=O)C12C</smiles><smiles>C[C@H]1CCC2=C(CO)C(=O)O[C@@H]2[C@@]2(C)C(=O)C=C[C@]12O</smiles>

(B)<smiles>C=C1C(=O)O[C@H]2C1CCC(C)C1(O)C=C(CO[Tl])C(=O)C21C</smiles>

$\mathrm{H}^{\prime}$

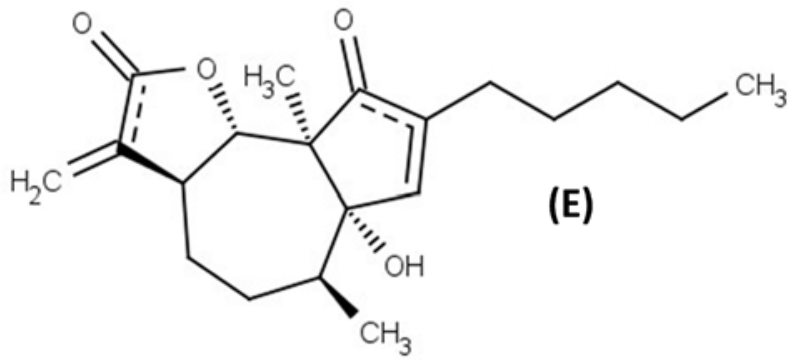

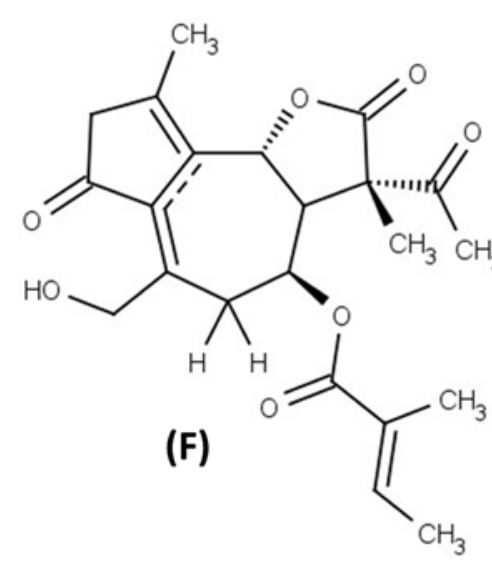

(D)<smiles>C=C1C(=O)O[C@H]2[C@@H]1CC[C@H](C)[C@@]1(O)C=C(CCC)C(=O)[C@@]21C</smiles>

Figure 6: Chemical structure of the lead compounds. (A) AC1MRFOP (CID3467446) (B) (6R, 6aS, 9bR)-6a-hydroxy-3(hydroxymethyl)-6, 9a-dimethyl-4H, 5H, 6H, 9bH-azuleno[4, 5-b]furan-2,9-dione (C) SCHEMBL4058769 (D) (3S, 3aS, 4S, 4aS, 7aR, 8S, 9aS)-3, 4a, 8-trimethyl-2, 5-dioxo-3H, 3aH, 4H, 7aH, 8H, 9H, 9aH-azuleno[6, 5-b]furan-4- yl (3S)-3-hydroxy-2-methylidenebutanoate (E) (3aS, 6S, 6aS, 9aR, 9bS)-6a-hydroxy-6, 9a-dimethyl-3-methylidene-8-pentyl-2H, 3H, 3aH, 4H, 5H, 6H, 6aH, 9H, 9aH, 9bH-azuleno[4, 5-b]furan-2,9-dione (F) (3S, 4S, 9bR)-3-acetyl-6-(hydroxymethyl)-3, 9- dimethyl-2, 7-dioxo-2H, 3H, 3aH, 4H, 5H, 7H, $8 \mathrm{H}, 9 \mathrm{bH}$ - azuleno[4, 5-b]furan-4-yl (2E)-2-methylbut-2-enoate (G) (3aR, 6S, 6aS, 9aR, 9bR)-8-heptyl-6a-hydroxy-6, 9a-dimethyl3-methylidene-2H, 3H, 3aH, $4 \mathrm{H}, 5 \mathrm{H}, 6 \mathrm{H}, 6 \mathrm{aH}, 9 \mathrm{H}, 9 \mathrm{aH}, 9 \mathrm{bH}$-azuleno[4, 5-b]furan-2,9-dione

Structural analysis of docked complexes of selected parthenin like compounds with Plasmodium vivax DHFR revealed strong chances of involvement of residues like Arg115, Leu136, Lys138, and Gly175 in inhibition. In addition to amino acids Leu136, Ile155, and Val178 appeared in the hydrophobic interactions. Moreover, the active site of human DHFR comprises of mostly hydrophobic amino acids as Ile7, Ala9, Trp24, Phe31, Phe34, Thr56, Pro61, and Ile60, Val115 and these amino acid residues are involved in strong hydrophobic interactions with the parthenin like compounds. The obtained results are expected to be useful for understanding not only the mode of inhibition but also in rapid and accurate prediction of the newly designed inhibitors. We also conclude that hydrophobic forces might play a highly influencing role in inhibition of Plasmodium vivax DHFR and successfully delineate specific functional groups that might be reSsponsible for hydrophobic effect of parthenin like compounds.

\section{ACKNOWLEDGEMENT}

PS and PPK acknowledge Indian Council of Medical Research (ICMR), India and UGC-CSIR, India respectively for providing the financial assistance in the form of Senior and Junior Research Fellowship. SK acknowledges Central University of Punjab for providing infrastructure facilities.

\section{Conflicting Interest}

The Authors declare no conflict of interest. 


\section{ABBREVIATIONS USED}

DHFR: Dihydrofolate reductase; DHF: Dihydrofolate ; dUMP: Deoxyuridine monophosphate; dTMP: Deoxythymidine monophosphate; THF: Tetrahydro folate; NADP+: Nicotinamide adenine dinucleotide phosphate; NADPH: Nicotinamide adenine dinucleotide phosphate, reduced; PDB: The Protein Data Bank; ADME/T: Absorption, Distribution, Metabolism, Excretion and Toxicity.

\section{REFERENCES}

1. WHO. World Malaria Report 2011. Geneva. 2011. World Health Organ

2. Qidwai T, Jamal F, and Khan MY, Sharma B. Exploring drug targets in isoprenoid biosynthetic pathway for plasmodium falciparum. Biochem Res Int. 2014; 1-12.

3. Chen C. Development of antimalarial drugs and their application in China: a historical review. Infect Dis Poverty. 2014; 3(1):9.

4. Olliaro PL, Yuthavong Y. An overview of chemotherapeutic targets for antimalarial drug discovery. Pharmacol. Ther. 1999; 81(2):91-110.

5. Thakkar SS, Thakor P, Doshi H, Ray A. 1, 2, 4-triazole and 1, 3, 4-oxadiazole analogues: synthesis, MO studies, in silico molecular docking studies, antimalarial as DHFR inhibitor and antimicrobial activities. Bioorg. Med. Chem. 2017; 25(15):4064-4075.

6. Patel TS, Vanparia SF, Patel UH, Dixit RB, Chudasama CJ, Patel BD, et al,. Novel 2, 3-disubstituted quinazoline-4(3H)-one molecules derived from amino acid linked sulphonamide as a potent malarial antifolates for DHFR inhibition. Eur J Med Chem. 2017; 129:251-265.

7. Kumar S, Pandey S, Pandey AK. In vitro antibacterial, antioxidant, and cytotoxic activities of Parthenium hysterophorus and characterization of extracts by LC-MS analysis. BioMed Res. Int. 2014; 2014:1-10.

8. Kapetanovic IM. Computer-aided drug discovery and development (CADDD): in silico-chemico-biological approach. Chem. Biol. Interact. 2008; 171(2):165-76.

9. Singh P, Singh RS, Rani A, Bast F. Homology modeling of chemokine CCR7, molecular docking, and in vitro studies evidenced plausible immunotherapeutic anticancer natural compounds. Med Chem Res. 2016; 25(10):2410-2424
10. Kongsaeree P, KhongsukP, Leartsakulpanich U, Chitnumsub P, Tarnchompoo B, Walkinshaw MD, et al,. Crystal structure of dihydrofolate reductase from Plasmodium vivax: pyrimethamine displacement linked with mutationinduced resistance. Proc. Natl. Acad. Sci. U.S.A. 2005; 102(37):13046-51.

11. Klon AE, Heroux A, Ross LJ, Pathak V, Johnson CA, Piper JR, et al, . Atomic structures of human dihydrofolate reductase complexed with NADPH and two lipophilic antifolates at $1.09 \AA$ and $1.05 \AA$ resolution. J. Mol. Biol. 2002; 320(3):677-93.

12. Friesner RA, Banks JL, Murphy RB, Halgren TA, Klicic JJ, Mainz DT, et al,. Glide: a new approach for rapid, accurate docking and scoring. 1. Method and assessment of docking accuracy. J. Med. Chem. 2004; 47(7):1739-49.

13. Jorgensen WL, Maxwell DS, Tirado-Rives J. Development and testing of the OPLS all-atom force field on conformational energetics and properties of organic liquids. J. Am. Chem. Soc. 1996; 118(45):11225-36.

14. Jorgensen WL, Tirado-Rives J. The OPLS [optimized potentials for liquid simulations] potential functions for proteins, energy minimizations for crystals of cyclic peptides and crambin. J. Am. Chem. Soc. 1988; 110(6):1657-66.

15. Shivakumar D, Williams J, Wu Y, Damm W, Shelley J, Sherman W. Prediction of absolute solvation free energies using molecular dynamics free energy perturbation and the OPLS force field. J. Chem. Theory Comput. 2010; 6(5):1509-19.

16. Jorgensen WL, Duffy EM. Prediction of drug solubility from structure. Adv. Drug Deliv. Rev. 2002; 54(3):355-66.

17. Lu JJ, Crimin K, Goodwin JT, Crivori P, Orrenius C, Xing L, et al,. Influence of molecular flexibility and polar surface area metrics on oral bioavailability in the rat. J. Med. Chem. 2004; 47(24):6104-7.

18. Tonelli M, Naesens L, Gazzarrini S, Santucci M, Cichero E, Tasso B, et al.. Host dihydrofolate reductase (DHFR)-directed cycloguanil analogues endowed with activity against influenza virus and respiratory syncytial virus. Eur J Med Chem. 2017; 135:467-78.

19. Srivastava V, Kumar A, Mishra BN, Siddiqi MI. Molecular docking studies on DMDP derivatives as human DHFR inhibitors. Bioinformation. 2008; 3(4):180-8.

20. Viira B, Gendron T, Lanfranchi DA, Cojean S, Horvath D, Marcou G, et al,. In silico mining for antimalarial structure-activity knowledge and discovery of novel antimalarial curcuminoids. Molecules. 2016; 21(7): 853-71.

21. Di L, Kerns EH. Drug-like properties: concepts, structure design and methods from ADME to toxicity optimization. Academic Press. 2015.

22. Veber DF, Johnson SR, Cheng HY, Smith BR, Ward KW, Kopple KD. Molecular properties that influence the oral bioavailability of drug candidates. J. Med. Chem. 2002; 45(12):2615-23.

23. Becker S, Groner B, Muller CW. Three-dimensional structure of the Stat3beta homodimer bound to DNA. Nature. 1998; 394(6689):145-51.

\section{PICTORIAL ABSTRACT}

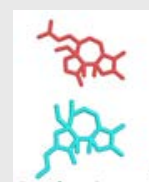

Parthenin

Like compounds
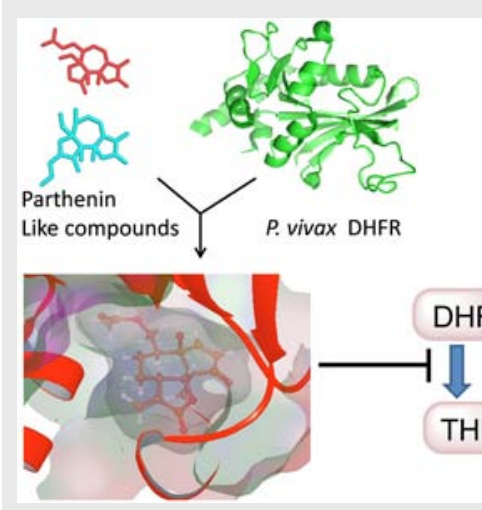

\section{SUMMARY}

- Parthenin like compounds has the ability to bind selectively with $P$. vivax dihydrofolate reductase protein. In comparison to known inhibitors (Dock Score up to -8.04), parthenin like compounds showed better Dock Score (up to -9.3). Arg 115, Leu 136, Lys 138, Gly 175, Ser 117, GIn 177 amino acid residues of $P$. vivax dihydrofolate reductase protein are involved in strong interactions with lead compounds. All the bestdocked lead compounds followed Lipinski's rule of five and showed drug-likeness, non-toxic, nonmutagenic and better biological properties. Thus parthenin like compounds showed possibilities to become potent anti-malarial agents of natural origin. Further in vitro and in vivo studies are required to study their potential. 


\section{About Authors}

Dr. Shashank Kumar: Obtained his B.Sc., M.Sc., and PhD. in Biochemistry from the University of Allahabad, Uttar Pradesh, India. He is working as Assistant Professor in the Department of Biochemistry and Microbial Sciences, Central University of Punjab, Bathinda, Punjab, India.

Dr. Pushpendra Singh: Dr. Singh did his B.Sc. and M.Sc. in Biotechnology from Awadhesh Pratap Singh University Rewa (M.P), India. He did his M.Phil. and PhD. in Biosciences, from Central University of Punjab, Bathinda, Punjab. Currently, he is working as ICMR, Research Associate at Tumor Biology Laboratory, National Institute of Pathology (ICMR), New Delhi, India.

Mr. Prem Prakash Kushwaha: Obtained his B.Sc. and M.Sc. Biochemistry from the University of Allahabad, Uttar Pradesh, India. He is pursuing Ph.D. from Central University of Punjab, Bathinda, Punjab, India.

Cite this article: Pushpendra S, Prem KP, Kumar S. Novel Potent Inhibitors of Plasmodium vivax Dihydrofolate Reductase: An in silico Antimalarial Drug Discovery. Indian $\mathrm{J}$ of Pharmaceutical Education and Research. 2018;52(1):122-34. 